\title{
NAUFRAGIOS COMUNICATIVOS EN \\ EL NUEVO MUNDO DESCUBIERTO POR CRISTÓBAL COLÓN: \\ UNA APROXIMACIÓN DIDÁCTICA \\ A LA MEDIACIÓN INTERCULTURAL \\ Mercedes Ariza \\ Universidad de Macerata (Italia)
}

\section{ABSTRACT}

This paper presents some reflections that emerged from a research project developed in the Department of Interdisciplinary Studies in Translation, Languages and Cultures (SITLeC) of the University of Bologna. During an eight-month workshop, a group of Italian students - beginners in Spanish - engaged in some extracurricular activities, resulting in the public performance of the play El Nuevo Mundo descubierto por Cristóbal Colón written by Lope de Vega. Students analysed the play from a linguistic and (inter)cultural perspective, giving special emphasis to the discovery of the other, the encounter of the Old and the New World and the miscommunication caused by lack of understanding in intercultural situations. All these aspects helped students to explore a range of verbal and nonverbal communicative strategies, as well as to improve their intercultural communicative competence and develop empathy. The in-depth analysis of the play showed students that the real shipwreck in intercultural encounters is incommunicability itself.

KEYWORDS: intercultural mediation, theatre, verbal and nonverbal communication

\section{RESUMEN}

El presente trabajo recoge algunas consideraciones sobre la importancia de la mediación intercultural a partir de un proyecto de investigación desarrollado en el Departamento de Estudios Interdisciplinarios sobre Traducción, Lenguas y Culturas (SITLeC) de la Universidad de Bolonia. Durante ocho meses un grupo de estudiantes italianos - la mayoría principiantes absolutos de español- analizaron la comedia de Lope de Vega El Nuevo Mundo descubierto por Cristóbal Colón y participaron en su montaje final. En particular, los estudiantes profundizaron en el análisis lingüístico e intercultural de la pieza, prestando una atención especial al descubrimiento del otro, al encuentro entre el Viejo y el Nuevo Mundo y a los malentendidos que pueden 
surgir cuando no se comprenden algunas cuestiones interculturales. Todos estos aspectos obligaron a los estudiantes a explorar diferentes estrategias de tipo verbal y no verbal con el objetivo de afianzar su competencia comunicativa intercultural y desarrollar la empatía.

PALABRAS CLAVE: mediación intercultural, teatro, comunicación verbal y no verbal

\section{INTRODUCCIÓN}

En el marco del proyecto de investigación "La formación de mediadores lingüísticos y la adquisición de la competencia comunicativa intercultural: el teatro en la enseñanza de las lenguas extranjeras, de la traducción y de la interpretación" (Departamento SITLeC, Universidad de Bolonia, sede de Forli ${ }^{1}$, explicaremos las motivaciones que nos llevaron a elegir la famosa comedia de Lope de Vega El Nuevo Mundo descubierto por Cristóbal Colón (Nuevo Mundo) para investigar sobre los escollos de la comunicación y la importancia de la mediación intercultural.

Durante el curso 2004-2005 el armonioso verso castellano y la osadía del descubridor atrajeron al escenario a un grupo de jóvenes universitarios italianos $^{2}$. Estos lectores-actores convivieron durante ocho meses con el genio de Lope, quien los llevó a percibir, sentir, comprender, interpretar y traducir la emoción del indio de Guanahamí bajo su propia piel. Durante esta larga travesía nuestros estudiantes y futuros mediadores lingüísticos aprendieron a descubrir al otro y a convivir con él y pudieron reflexionar sobre las destrezas y habilidades necesarias para adquirir una sólida competencia comunicativa intercultural.

\footnotetext{
${ }^{1}$ El proyecto, ideado y coordinado por María Isabel Fernández García, ha sido financiado por la Universidad de Bolonia. Para una detallada descripción, véase Fernández García y Biscu (2005/2006; 2008).

2 Luciano Baldan, Carla Bondì, Chiara Castagnini, Renee Castiglione, Cinzia Catana, Dalila Crobu, Mara De Col, Valeria Di Giacomo, Mirella Facchinetti, Abdelatif Faouzi Hakim, Elisa Fusconi, Federica Mazzocconi, Chadi Monti, Letizia Montroni, Adele Paolicelli, Luana Rupi, Lucia Zardi. Los estudiantes, la mayoría principiantes absolutos de español, pertenecen a la Facultad de Traducción e Interpretación de la Universidad de Bolonia, sede de Forlì. Coordinación del aula de teatro: Claudio Bendazzoli, Maria Giovanna Biscu e Isabel Fernández con la autora del trabajo. Para la historia de nuestra experiencia de teatro universitario, véase Fernández García et al. (2007a, 2007b).
} 
En este trabajo analizaremos algunos de los problemas comunicativos y malentendidos (inter)culturales que nuestros actores-mediadores tuvieron que resolver antes del desembarco final ${ }^{3}$.

\section{EL NUEVO MUNDO Y LA ADQUISICIÓN DE LA COMPETENCIA COMUNICATIVA INTERCULTURAL}

En nuestro proyecto de investigación (Fernández García y Biscu, 2005/2006; 2008), la comedia de Lope encajaba perfectamente con nuestro objetivo didáctico: sensibilizar a los estudiantes acerca de la adquisición de la competencia comunicativa intercultural. Por un lado, narraba el primer "choque cultural" de la historia (Rodrigo Alsina 1999: 69-79) y permitía reflexionar acerca de los malentendidos que pueden derivar del etnocentrismo y del contraste entre lengua y cultura (Rodrigo Alsina, 1999: 69-79; Oliveras Vilaseca, 2000: 71). Por otro, nos ofrecía un gran abanico de situaciones para analizar las competencias y habilidades necesarias para aprender a "descubrir al otro" y desarrollar la empatía, esto es, la capacidad de sentir la emoción ajena (Rodrigo Alsina, 1999: 79).

A su vez, esta pieza nos resultó de candente actualidad y resultaba paradigmática de la alteridad: para comprender y entrar en contacto con el otro, los estudiantes comprobaron en carne propia la necesidad de descentrarse de su propia cultura (Byram y Fleming, 2001: 168). Finalmente, zambullir a nuestros futuros mediadores lingǘsticos en un texto colonial como Nuevo Mundo nos permitía dar una respuesta teatral a la pregunta que dio origen a nuestro proyecto de investigación ("la competencia comunicativa intercultural, ¿se puede enseñar/aprender?” [Fernández García y Biscu, 2005/2006: 5]).

\section{NAUFRAGIOS COMUNICATIVOS: NUESTRA EXPLOTACIÓN DIDÁCTICA}

Los versos de Lope, con su mirada ácida e irónica, arrojaron a nuestros estudiantes ante la fragilidad de la comunicación humana y, en particular, ante los problemas comunicativos que pueden surgir cuando no se comparten los mismos paradigmas lingǘsticos y culturales del otro. En Nuevo Mundo

\footnotetext{
${ }^{3}$ La puesta en escena se presentó en el Cantiere Internazionale Teatro Giovani 2005 VI edizione, una manifestación cultural organizada por el Ayuntamiento de Forlì, el Centro de Estudios Teatrales del "Dipartimento di Studi Interdisplinari di Traduzione, Lingue e Cultura" de la Universidad de Bolonia (sede de Forli), la Universidad de Harvard, el DAMS de la Universidad de Bolonia, la Universidad de Columbia. Para el análisis de nuestra travesía teatral, véase Ariza, Biscu y Fernández García (2007).
} 
interpretar la realidad ajena desde la propia visión del mundo provoca profundos malentendidos y la falta de diálogo con el otro, como veremos más adelante, lleva al naufragio de todo intento de comunicación.

En su comedia, Lope elige presentarnos la invasión de los españoles y, por tanto, la interpretación de un mundo ajeno a través de la mirada indígena. Los naufragios comunicativos empiezan en el segundo acto cuando el indio Auté avista las carabelas entre brumas y celajes. Según su narración fantástica (vv. 1479-1520)4, las embarcaciones son casas que caminan sobre las aguas, las carnes de los hombres que las habitan son de colores, las escopetas son palos de los que salen llamas y humo ${ }^{5}$. Tras esta interpretación, los dos caciques proponen las suyas, aún más delirantes. Dulcanquellín incluso reprende a Auté, afirmando que no se trata de casas sino de peces ("Ignorante, ¿qué dices? / peces son, peces que braman que andando por esas islas / a hartarse de carne humana,/se han comido aquesos hombres / que a voces sus dioses llaman", vv. 1521-1525). Tapirazú, para no ser menos, inventa desde la tradición una respuesta a lo desconocido, rememorando los cuentos de su abuelo: "Yo sé mejor lo que ha sido, / que estas son reliquias claras / de los gigantes que un tiempo / vinieron a estas montañas" (vv. 1531-1534). Evidentemente, los indios perciben la novedad absoluta de su visión, pero no pueden comprenderla y, por lo tanto, no les queda más remedio que interpretarla desde sus propios paradigmas.

Otro caso emblemático se produce cuando los indios entran en contacto por primera vez con la religión cristiana. Ante la cruz plantada en la playa por los españoles cada personaje propone su interpretación del objeto misterioso. Según Dulcanquellín, el madero sirve para: "para meter a la tierra/ las casas desde la mar" (vv. 1758-1759). Tapirazú, por su parte, explica: "sin duda que es atalaya /para subirse sobre ella" (vv. 1774-1775). Sin embargo, Tacuana intuye "que debe de ser /alguna sagrada cosa" (vv. 1770-1771) y, más adelante exclamará: "Palo santo, palo hermoso,/ Dios en ti no conocido [...] no nos mates por tu ofensa,/que ya todos te adoramos" (vv. 1848-1853) ${ }^{6}$.

Desde un punto de vista didáctico el análisis de estos malentendidos fue fundamental para los estudiantes. En primer lugar, para poder conocer al otro y convivir con él, nuestros futuros mediadores lingüísticos se vieron obligados a abandonar su propia visión del mundo, poniendo en tela de juicio

\footnotetext{
${ }^{4}$ Seguimos la edición de Jean Lemartinel y Charles Minguet (1980). Todas las citas se referirán a esta edición.

5 Tal como destaca Kirschner (1993: 54-55), estas escenas son emblemáticas, puesto que "la descripción de la llegada de las tres carabelas está ya imbuida por la naciente mitología que surgirá alrededor de la imagen que el indio se hará del español y la que el español se hará del indio".

${ }^{6}$ Sorprende constatar cómo el indio de Lope "desde su inocencia adánica será capaz de intuir y comunicar - inconscientemente - los misterios más profundos de la fe cristiana” (Bradley 2004).
} 
aquellos modelos culturales con los que habían crecido (Balboni, 1999: 17). En segundo lugar, pudieron comprobar que los choques culturales y malentendidos no están relacionados exclusivamente con el dominio lingüístico sino también con el desconocimiento de aspectos socioculturales. Al respecto, Paolo Balboni (1999: 39-49), en su libro-guía en torno a la comunicación intercultural, nos recuerda que estamos tan empapados en/por nuestra cultura que no reflexionamos sobre algunos elementos culturales básicos que influyen en nuestra manera de comunicar.

A su vez, nuestros estudiantes pudieron constatar que para interpretar un acto comunicativo es imposible trascender del carácter triádico de la comunicación, es decir, "el continuo verbal-paralingüístico-kinésico formado por sonidos, silencios y por movimientos y por posiciones estáticas" (Poyatos 1994: 130). A lo largo de su travesía, pues, nuestros estudiantes/actores se vieron obligados a considerar también la conducta no verbal como fuente imprescindible de comunicación. Es más, descubrieron en carne propia cómo el ruido del silencio, la intensidad de una mirada o la espontaneidad de un gesto pueden comunicar más que una palabra.

\subsection{Palca, mediadora entre EL Viejo y EL Nuevo Mundo7 ${ }^{7}$}

El abismo de la incomunicación parece todavía más insondable en la primera toma de contacto entre españoles y nativos, en la que se observa una compleja y divertida fusión de señas, gestos y lengua ${ }^{8}$. Por las señas de los marineros, Palca intuye que le están preguntando cómo se llama y, cuando responde, desencadena una serie de juegos interpretativos y lingüísticos encaminados a provocar la risa en el público. Como vemos en el siguiente ejemplo, el verso dilata el efecto cómico de las réplicas, encadenando las risas con las rimas:

$\begin{array}{ll}\text { PALCA. } & \begin{array}{l}\text { Señas hacen, si mi nombre } \\ \text { preguntan, responder quiero. } \\ \\ \text { ¡Palca, Palca! }\end{array} \\ \text { COLÓN. } & \begin{array}{l}\text { Lo primero } \\ \text { dice Palca. }\end{array} \\ \text { BARTOLOMÉ. } & \text { ¿Es rey, es hombre? }\end{array}$

\footnotetext{
7 Para un análisis del papel del indio como mediador entre dos culturas, véanse: "El intermediario" en Greenblatt (1994: 203-248); "Los intérpretes” en Martinell Gifre (1988: 5999); "Colon et les Indiens" en Todorov (1982: 40-55); "Las palabras perdidas de La Malinche. El mito de la comunicación y el fallo comunicativo" en Restall (2004: 123-149).

8 Recordemos que a la crítica no le ha pasado desapercibido el simulacro teatral que les permite a los indios dialogar tranquilamente en verso castellano antes de ser descubiertos (Menéndez Pelayo 1949: 310).
} 


$\begin{array}{ll}\text { PALCA. } & \begin{array}{l}\text { El señor pregunta, en fin. } \\ \text { Cacique, Dulcanquellín. }\end{array} \\ \text { BARTOLOMÉ. } & \text { Cocique debe de ser, } \\ & \text { que al fin es bárbara lengua. } \\ & \text { que habrá adentro que comer, } \\ & \text { y Dulcán, que no habrá mengua. } \\ & \text { Y por ventura Quellín, } \\ & \text { será el pan, o será el vino. } \\ & \text { ¡Vino aquí, qué desatino! } \\ \text { COLÓN. } & \text { (Ved qué gentil Candía o Rin! } \\ & \text { (vvint-1673) }\end{array}$

Entre bromas y veras, Lope de Vega resulta un maestro para nuestros futuros mediadores. A través de estas réplicas nuestros estudiantes no solo pudieron reflexionar, una vez más, acerca de los malentendidos y del "carácter triádico de la comunicación" (Poyatos 1994) sino también acerca de un problema teórico fundamental en sus estudios: la intraducibilidad. El término 'cacique' condensa esta cuestión y, como pudimos ver, la solución radica en cultivar con paciencia el diálogo. Un siglo después de la conquista, no entender esta palabra tiene consecuencias tragicómicas: Bartolomé inventa lo que su imaginación desea, pan y vino.

Para subrayar el efecto cómico que surge de este malentendido, en nuestra puesta en escena optamos por modificar los tipos de vino presentes en el texto original ("Candía” y "Rin") con "Porto" y "Lambrusquín". El primero para remitir al país de donde zarparon Colón y su tripulación y el segundo para hacer alusión al Lambrusco, un vino típico de la región italiana de Emilia Romaña de donde procedía nuestro público?.

A lo largo de la obra, el tema de la comida y de la hospitalidad ofrece nuevos ejemplos de fracasos comunicativos. Al final del segundo acto, Colón le pregunta a Dulcanquellín: “¿Habrá que comer?”; el cacique hipotiza: “Sospecho / que nos piden que comer" (vv. 2002-2003). Dulcán, para demostrar que quiere recibir a sus huéspedes con todos los honores, manda preparar un banquete a base de criados asados ${ }^{10}$ :

DULCANQUELLÍN. Mata, Auté, cuatro criados de los más gordos que hallares, y entre silvestres manjares

\footnotetext{
${ }^{9}$ No olvidemos que sólo una inmensa minoría de nuestro amable público forlivés comprendía el armonioso discurrir del verso castellano.

10 Tal como nos recuerda Shannon (1989: 75-76), en más de una ocasión, Lope recurre humorísticamente al paradigma del canibalismo.
} 


\subsubsection{El uso del cuerpo}

En cuanto al alfabeto de señas, gestos y miradas que dio lugar a las primeras tentativas de diálogo intercultural en Nuevo Mundo, explicaremos de qué manera la conducta no verbal sustituyó las palabras sobre todo al principio del descubrimiento, cuando los españoles y los indígenas, ante la imposibilidad de establecer un contacto verbal con el otro, optaron por un gesto o un movimiento para comunicar.

Recordamos que una parte fundamental de nuestro trabajo se centró en el análisis de material iconográfico y artístico de las culturas precolombinas. Durante esta fase de auto-observación, una de las más hermosas desde un punto de vista teatral y emotivo, nuestros estudiantes lograron imaginar el gesto indio del que nacían las palabras y, con este, percibir y sentir la realidad india. Además, consiguieron desarrollar una lengua propia que les permitía comprender al otro y entrar en contacto con él y con su cultura (Ariza, Biscu, Fernández 2007).

En el ámbito del comportamiento kinésico (Knapp 1980; Poyatos 1994), los españoles recurrieron a "emblemas", esto es, gestos sin palabras que describen acciones comunes a la especie humana y parecen trascender de una cultura particular (Knapp 1980: 17-20), como en el siguiente ejemplo, donde encontramos el emblema de comer (llevarse la mano hacia la boca) ${ }^{11}$ :

$\begin{array}{ll}\text { ARANA. } & \begin{array}{l}\text { Ea, pues adentro mira, } \\ \text { que comer hay, ¿̇o es ansí? }\end{array} \\ \text { PALCA. } & \text { A la boca ha señalado, } \\ & \text { comer pide, si mandioca, cazaví... } \\ \text { PINZÓN. } & \text { Mostró la boca. } \\ \text { BARTOLOMÉ. } & \text { Y aun el hígado ha mostrado. } \\ & \text { Sin duda habrá que comer. } \\ & \text { (vv. 1680-1686) }\end{array}$

Para averiguar sobre la existencia de nuevas tierras se recurrió a gestos señalativos, dando lugar a una repetición del comportamiento verbal (Knapp 1980: 27), como vemos en los siguientes versos:

BARTOLOMÉ. ¿Cómo se llama esta tierra?

DULCANQUELLíN. Guanahamí, Guanahamí.

\footnotetext{
11 Se trata de un caso de realización sólo kinésica. Véanse las diez realizaciones de lenguaje, paralenguaje y kinésica en Poyatos (1994: 144-147).
} 
COLÓN. ¿ ¿Hay tierra más adelante?

DULCANQUELLÍN. Barucoa, Barucoa.

(vv. 1952-1953)

Por su parte, la india Palca se muestra, una vez más, capaz de interpretar las señas de los españoles y en su afán por dejar claro el nombre de su cacique, recurre al uso de "ilustradores", es decir, actos no verbales que sirven para ilustrar lo que se dice verbalmente y que se usan intencionadamente para ayudar a la comunicación (Knapp 1980: 20), como notamos a continuación:

PALCA. Creo que me han preguntado
si hay a caso otro señor,
en esa isla mayor,
las señas lo han declarado.
Quiéroles decir que sí,
Tapirazú, Tapira...
(vv. $1675-1680)$

En cuanto a nuestro montaje, la estudiante-mediadora-india Palca se ensimismó tanto en su papel de intermediaria que improvisó en el escenario una clase de fonética india, puntuando las sílabas TA-PI-RA-ZÚ con evidentes gestos de la mano.

En su comedia, Lope de Vega rescata a sus personajes a través del humor, del amor y de la mediación. La india Palca, tras el temor inicial, parece comunicarse perfectamente con los españoles, intentando interpretar los signos verbales y no verbales de esos "hombres hermosos". Sin embargo, en una escena sucesiva, cuando ella va a llamar a su gente para explicarles lo que ha pasado, declara que los españoles no le han hablado:

$\begin{array}{ll}\begin{array}{l}\text { DULCANQUELLÍN. } \\ \text { PALCA. }\end{array} & \begin{array}{c}\text { Palca, ¿habláronte? } \\ \text { Pues no. }\end{array} \\ \text { DULCANQUELLÍN. } & \text { ¿Qué entendiste? } \\ \text { PALCA. } & \text { Que querían } \\ & \text { comer, y que aquí os traían } \\ & \text { de esto que os mostrase yo. } \\ & (\text { vv. 1894-1896) }\end{array}$

Palca logra comprender a los invasores aunque sugiere que en el momento clave del primer encuentro entre Viejo y Nuevo Mundo no hubo comunicación, es decir, traducción. Enseguida, la comicidad de su declaración deja espacio a una amarga constatación: una vez más asistimos a la 
incertidumbre de la comunicación, a ese "antimito del fallo comunicativo" del que habla Restall (Restall 2004: 132). Sin embargo, en nuestra propuesta dramatúrgica Palca nos deja la esperanza de que, al final, incluso mundos radicalmente diferentes se pueden encontrar (Ariza, Biscu, Fernández 2007).

Finalmente, tal como nos recuerda Martinell Gifre (1988: 28), los gestos que utilizaron los españoles durante sus primeras tentativas de diálogo, trascendían de su mera función indicadora y localizadora, puesto que servían para transmitir órdenes e imponerse ante los nativos:

FRAY BUYL. Anda, ve y llama más gente, dalde más sartas que dar.

(vv. 1706-1707)

TERRAZAS. Ve, amigo, y trae de esto alguno.

(v. 1982)

\subsubsection{El papel de los regalos}

Tras el desconcierto inicial, Palca se comunica con los españoles y aprecia su "calor y blandura", demostrando una vez más que los paradigmas son flexibles y que la comunicación entre los dos mundos es posible gracias al diálogo y a la mediación. Sin embargo, cuando acepta con inocencia las dádivas de los europeos y se mira en el espejo regalado -objeto extraño y extranjeroPalca retorna al temor, ya que en ese cristal se refleja no sólo su rostro sino todo el dilema de la alteridad:

\begin{tabular}{|c|c|}
\hline COLÓN. & $\begin{array}{l}\text { Ya entiende, un espejo muestra, } \\
\text { dádsele, y un cascabel, } \\
\text { toma, y miraraste en él. } \\
{[\ldots]}\end{array}$ \\
\hline PALCA. & ¡Ay! \\
\hline COLÓN. & $\begin{array}{l}\text { De velle se retira, } \\
\text { paso que se espanta mas. } \\
\text { ¡Tenla, que huye! }\end{array}$ \\
\hline PALCA. & $\begin{array}{l}\text { ¡Ay de mí, } \\
\text { otra Palca como yo } \\
\text { los cascabeles tomó! } \\
\text { (vv. 1691-1701) }\end{array}$ \\
\hline
\end{tabular}

El análisis de estos versos nos permitió introducir otro tema fundamental para el estudio de la comunicación intercultural: el ofrecimiento e intercambio de regalos. Según la taxonomía propuesta por Paolo Balboni 
(1999: 115-116) ${ }^{12}$, el uso de objetos con fines comunicativos es una válida herramienta de observación intercultural.

Durante los primeros contactos con los nativos, los españoles ofrecieron dádivas para predisponer a la paz y asegurar una situación no conflictiva. Según el interesante estudio de Martinell Gifre y Vallés (1997: 1336), los recién llegados conocían la eficacia de este tipo de conducta y, conscientes de la ingenuidad de los naturales, tan diversos por lengua y cultura, ofrecían presentes "para hacerlos vasallos” (Martinell Gifre, 1988: 54)13.

En nuestra comedia, los españoles procuraron favorecer el diálogo ofreciendo espejos, cascabeles y cuentas de vidrio; "cosillas" que ilusionaban a los nativos ("qué buen hado los conduce / adonde nadie ha llegado", vv. 19051906). Sin embargo, al margen de este "proceso de adentramiento y empatía" (Kirschner, 1993), los recién llegados embaucaban al nativo y lo preparaban al (des)engaño final:

$\begin{array}{lllll}\text { FRAY BUYL. } & \begin{array}{l}\text { ¡Anda, ve y llama más } \\ \text { dalde más sartas que dar. }\end{array} & & \text { gente, } \\ \text { PALCA. } & \begin{array}{l}\text { Que a otras vaya a } \\ \text { me dice, voy prestamente. }\end{array} & & \text { llamar } \\ \text { COLÓN. } & \begin{array}{l}\text { Mientras que la gente } \\ \text { saquemos las armas todas. }\end{array} & & \text { llama, }\end{array}$
(vv. 1706-1711)

Los indígenas, por su parte, daban lo que tenían con liberalidad y ofrecían “en señal de bienvenida” (Martinell Gifre y Vallés, 1997: 21). En más de una ocasión, sorprendían al español por su falta de avidez e incapacidad de calibrar el valor "real" de lo que recibían.

Para dejar de manifiesto esta diferente valoración de los objetos y de los intercambios, nuestros estudiantes-actores propusieron que en el montaje de Nuevo Mundo se dedicara especial atención al ritual de la boda indígena que abre el segundo acto de la comedia. La armonía del canto y baile, la "la divina hermosura" de Tacuana, los tesoros encerrados en esa "tierra dichosa y bella" y la serenidad de los rituales chocaron en el escenario con la avidez de los españoles, movidos por "una amistad fingida" cuya única finalidad era "robar oro y mujeres" (v. 2796) ${ }^{14}$.

\footnotetext{
12 Para la observación de la comunicación intercultural, Balboni (1999: 115-116) propone los siguientes elementos: a) valores culturales que interactúan con la comunicación; b) uso del cuerpo con fines comunicativos; c) uso de objetos con fines comunicativos; d) la lengua; e) acciones y situaciones comunicativas.

13 Según nos recuerda Arrom (1992: 35), los nativos se convertían en "el arquetipo perfecto del que siglos después habría de llamarse el buen salvaje.".

14 Además, tal como nos recuerda (Brotherton 1994: 36), la armonía inicial de la boda indígena presentada por Lope reflejaba la condición de los nativos antes de la llegada de los españoles.
} 


\section{CONSIDERACIONES FINALES}

A lo largo del presente trabajo intentamos reflexionar sobre los problemas comunicativos y malentendidos que pueden surgir cuando no se comparten los mismos paradigmas lingüísticos y culturales del otro. Nuestra lectura intercultural de Nuevo Mundo permitió sensibilizar a nuestros estudiantes/actores acerca de la importancia del diálogo y de la mediación frente a la fragilidad de la comunicación humana. Al respecto, nuestros futuros mediadores lingüísticos pudieron comprobar que para comunicar no solo se utilizan palabras sino también gestos, miradas y posturas. Es más, como hemos podido comprobar, el uso de objetos e intercambio de regalos con fines comunicativos es una válida herramienta de observación intercultural. Por todo ello, nuestros estudiantes se vieron obligados a descentrarse de sus valores y a desarrollar la empatía.

Por otro lado, confesamos que nuestra lectura de Nuevo Mundo proyectó en el escenario un nuestro íntimo deseo: ¿escribió Lope una obra sobre Colón descubridor a causa del silencio que desde 1577 azotaba las crónicas de Indias? ¿Acaso Lope quiso romper con esa visión unidimensional de la llegada-invasión-conquista-descubrimiento que concordaba con el horizonte de expectativas del público de la época? Al fin y al cabo, compartimos la opinión de ciertos estudiosos (Castells 2000; Shannon 1989) que ven en Nuevo Mundo una pieza paradigmática que ofrece una visión muy compleja de la expansión del imperio español y que pone en tela de juicio los dos motores del descubrimiento/conquista de América: la riqueza y la religión.

De lo que sí no cabe ninguna duda es que para nosotros y para nuestros estudiantes/ actores se trató de un nuevo descubrimiento del Nuevo Mundo. Un descubrimiento que nos llevó a descubrirnos a nosotros mismos con nuestras miserias y fragilidades: un espejo donde reflejar, antes que nada, nuestra alteridad.

\section{BIBLIOGRAFÍA}

Arrom, José Juan (1992). "En demanda de Cathay: lo real y lo imaginario en el Diario del primer viaje de Colón”. Cuadernos Americanos. Nueva Época. Año 6, vol. 3 [33], 31-47.

Ariza M., Biscu M. G. y Fernández García M. I. (2007): “The Madness of Inventing New Worlds". Scenario. Online Journal -ISSN 1649-8526. [en línea]: http://www.ucc.ie/en/scenario. [consulta: 25 de abril de 2009]

BAlBOni, Paolo (1999): Parole comuni culture diverse. Guida alla comunicazione interculturale. Venezia, Marsilio. 
BYRAM, M. Y M. FLEMING (2001): Perspectivas interculturales en el aprendiaje de idiomas. Enfoques a través del teatro y la etnografía. Madrid, Cambridge University Press.

BradLey, Nelson (2004): "La emblemática como marco teórico- crítico en los estudios transatlánticos" en Congreso Abierto. Publicación en la Red de las actas del 40 Congreso de la $A C H$. [en línea]: http://fis.ucalgary.ca/ACH/Congreso abierto/2004/Bradley_Nelson.htm [consulta: 02 de mayo de 2009]

Brotherton, John (1994): “Lope de Vega's El Nuevo Mundo descubierto por Cristóbal Colón: Convention and Ideology", Bulletin of The Comediantes, 46, 1 , 33-47.

Castells, Ricardo (2000): “Oro e idolatría en El nuevo mundo descubierto por Colón de Lope de Vega", Neophilologus, 84, 3, 385-397.

FERnÁndez GARCÍA, M. I Y M. G. BisCU (2005/2006): "Theatre in the Acquisition of Intercultural Communicative Competence: The Creation of a Multilingual Corpus of Dramatic Texts for the Training of Future Language Mediators", International Journal of Learning, 12, Issue 10, 327-335. [en línea]: http://www.Learning-Journal.com [consulta: 25 de abril de 2009] FERNÁNDEZ GARCÍA M. I. et al. (2007A): "El aula-escenario en la formación de futuros mediadores lingüísticos: un puerto para descubridores de la otredad", página web que presenta este proyecto trienal. Webmaster: Luciano Baldan. [en línea]: http://www.aulapalcoscenico.it [consulta: 20 de abril de 2009]

- et al. (2007B): "El aula-escenario en la formación de futuros mediadores lingüísticos: un puerto para descubridores de la otredad", Memorias del Simposio Internacional del 35 aniversario de la Institucionalización de los Estudios Superiores en Pinar del Rio. Del 2 al 4 de octubre de 2007. Congreso Internacional "Lengua y Cultura: tendiendo puentes a través de las artes". Facultad de Ciencias Sociales y Humanísticas, Universidad de Pinar del Río, Cuba. ISBN: 978959-16-0599-3.

- y M. G. BISCU (2008): "Intercultural Settings for Language Mediation: Evaluation of a Research Project on Language Mediator Training Through Theatre", International Journal of Learning, 15, 1, 51-63, [en línea]: http://www.Learning-Journal.com. [consulta: 20 de abril de 2009]

GreENBLATT, Stephen (1994): Meraviglia e possesso. Lo stupore di fronte al Nuovo Mondo. Bologna, Il Mulino.

Kirschner, Teresa (1993): "Exposición y subversión del discurso hegemónico en pro de la conquista en El Nuevo Mundo de Lope de Vega" en Campbell Y. (ed.): El escritor y la escena. Actas del I Congreso de la Asociación Internacional de Teatro Españoly Novohispano de los Siglos de Oro (18-21 de marzo de 1992, Ciudad Juárez), Universidad Autónoma de Ciudad Juárez, Ciudad Juárez, México, 45-58. 
KNAPP, Mark (1980): La comunicación no verbal. El cuerpo y el entorno. Barcelona, Paidós.

Lemartinel, Jean y C. Minguet (1980): El Nuevo Mundo descubierto por Cristóbal Colón. Comedia de Lope de Vega Carpio. Lille, PU de Lille.

MARTinell Gifre, Emma (1988): Aspectos lingüísticos del descubrimiento y de la conquista. Consejo Superior de Investigaciones Científicas. Madrid, Rascar.

- y N. VAlLÉS (1997): "Ellos vinieron a rescatar" en Zimmermann, K., Bierbach, C. (eds): Lenguaje y comunicación intercultural en el mundo hispánico. Frankfurt am Main, Verveurt, Madrid, Iberoamericana, 13-36.

Menéndez Pelayo, Marcelino (1949): "El Nuevo Mundo descubierto por Cristóbal Colón” en Menéndez Pelayo, Marcelino (ed.): Estudios sobre el teatro del Lope de Vega. Santander, Aldus, Vol. 34, 306-325.

Oliveras VILASECA, Angels (2000): Hacia la competencia intercultural en el aprendizaje de una lengua extranjera. Estudio del choque cultural y de los malentendidos. Madrid, Edinumen.

POYATOS, Fernando (1994): La comunicación no verbal I. Cultura, lenguaje y conservación. Madrid, Istmo.

Restall, Matthew (2004): Los siete mitos de la conquista española, trad. cast., Barcelona, Paidós.

Rodrigo AlsinA, Miquel (1999): Comunicación intercultural. Barcelona, Anthropos.

SHANNON M., Robert (1989): Visions of the New World in The Drama of Lope de $V$ ega. New York \& Bern, Peter Lang.

TODOROv, Tzvetan (1982): La conquête de l'Amérique. La question de l'autre. Paris, Editions du Seuil. 